\title{
Effect of dexamethasone on endothelial nitric oxide synthase in experimental congenital diaphragmatic hernia
}

\author{
Bruce O Okoye, Paul D Losty, Michael J Fisher, Irene Wilmott, David A Lloyd
}

\begin{abstract}
Aims-To study the effect of prenatal glucocorticoid treatment on endothelial nitric oxide synthase (eNOS) expression in rats with congenital diaphragmatic hernia (CDH).

Methods-CDH was induced in fetal rats by the maternal administration of nitrofen on day 9.5 of gestation. Dexamethasone was administered on days 18.5 and 19.5 before delivery of the fetuses on days 20.5 and 21.5. Pulmonary eNOS protein expression was studied by western immunoblotting and immunohistochemistry.

Results-On day 20.5, eNOS expression was significantly reduced in CDH pups compared with normal control rats. Dexamethasone treated CDH pups had eNOS concentrations equivalent to those of normal animals. By day 21.5, however, there was no detectable difference in eNOS expression between the experimental groups.

Conclusions-eNOS is deficient in near term (day 20.5) CDH rats. Dexamethasone restores eNOS expression in these animals to that seen in normal rat lungs. At term, the precise role of eNOS in the pathophysiology of $\mathrm{CDH}$ remains uncertain.
\end{abstract}

(Arch Dis Child Fetal Neonatal Ed 1998;78:F204-F208)

Keywords: congenital diaphragmatic hernia; endothelial nitric oxide synthase; glucocorticoids; nitrofen

Congenital diaphragmatic hernia $(\mathrm{CDH})$ continues to have an unacceptably high mortality due to the lethal combination of pulmonary hypoplasia and pulmonary hypertension. ${ }^{1}$ Pulmonary hypertension is characterised by persistent and severe hypoxia associated with ductal shunting from the pulmonary circuit to the systemic circulation. ${ }^{2}$ In $\mathrm{CDH}$ a combination of factors contribute to its occurrence. A reduced and excessively muscularised pulmonary vascular bed exhibits abnormal vascular reactivity, resulting in profound vasoconstriction and refractory hypoxaemia. ${ }^{2}$ Various treatments aimed at improving pulmonary hypertension have entailed ventilatory strategies such as hyperventilation and permissive hypercapnia, vasodilators, and extracorporeal membrane oxygenation (ECMO).$^{45}$

The mechanisms responsible for abnormal vascular reactivity in $\mathrm{CDH}$ are not completely understood. During fetal life, the pulmonary vasculature is a high pressure bed with minimal blood flow through the lungs. However, at birth, pulmonary vascular resistance decreases and blood flow increases as the lungs take over the function of gaseous exchange from the placenta. ${ }^{6}$ The pulmonary vascular endothelium is of vital importance in the perinatal regulation of pulmonary vascular tone and blood flow. ${ }^{7}$ It releases a host of vasodilator and vasoconstrictor substances which, acting in a paracrine manner, stimulate the contraction or relaxation of adjacent vascular smooth muscle cells. ${ }^{7}$ Nitric oxide (NO) is believed to have a pivotal role in the transition of the pulmonary circulation from fetal to neonatal life. ${ }^{8}$ A potent vasodilator, it is generated in the pulmonary vascular endothelium through the action of the enzyme, endothelial nitric oxide synthase (eNOS). ${ }^{9}{ }^{10} \mathrm{NO}$ diffuses into adjacent smooth muscle cells where it increases cellular concentrations of cyclic guanosine monophosphate thus producing vaso-relaxation. ${ }^{9}{ }^{10}$ As the pulmonary circulation in some neonates with $\mathrm{CDH}$ fails to adapt or sustain the physiological transition to extrauterine life, it has been suggested that NO may be deficient in $\mathrm{CDH}$. Inhaled NO treatment has been instituted as a specific pulmonary vasodilator in $\mathrm{CDH}$ patients as a means of treating pulmonary hypertension related crisis with varying clinical results. $^{11-17}$

Glucocorticoids have an important role in the regulation of normal lung development and exert profound maturational effects on the developing fetal lung. ${ }^{18}$ Antenatal corticosteroid treatment is now well established as a means of preventing respiratory distress syndrome in premature newborns. ${ }^{19}$ In experimental CDH animal models, antenatal glucocorticoids improve surfactant biochemical immaturity, increase lung compliance, and enhance lung morphology. ${ }^{18-23}$ The effect of this pharmacological intervention on the prevention or treatment of pulmonary hypertension in $\mathrm{CDH}$ remains unknown. The aim of the present study was to evaluate the effect of antenatal glucocorticoids on eNOS expression as a marker of pulmonary vascular reactivity in the lungs of rats with congenital diaphragmatic hernia induced by nitrofen.

\section{Methods}

CREATION AND TREATMENT OF CDH

Timed pregnant Sprague-Dawley rats (Charles River UK, Ltd) (vaginal plug positive $=$ day 0 ) were given $100 \mathrm{mg}$ nitrofen (Zhejiang Chemicals, China) by gavage on day 9.5 of gestation (term = day 22) to induce left sided $\mathrm{CDH}$ in fetal rats. ${ }^{24}$ Control animals received olive oil. 


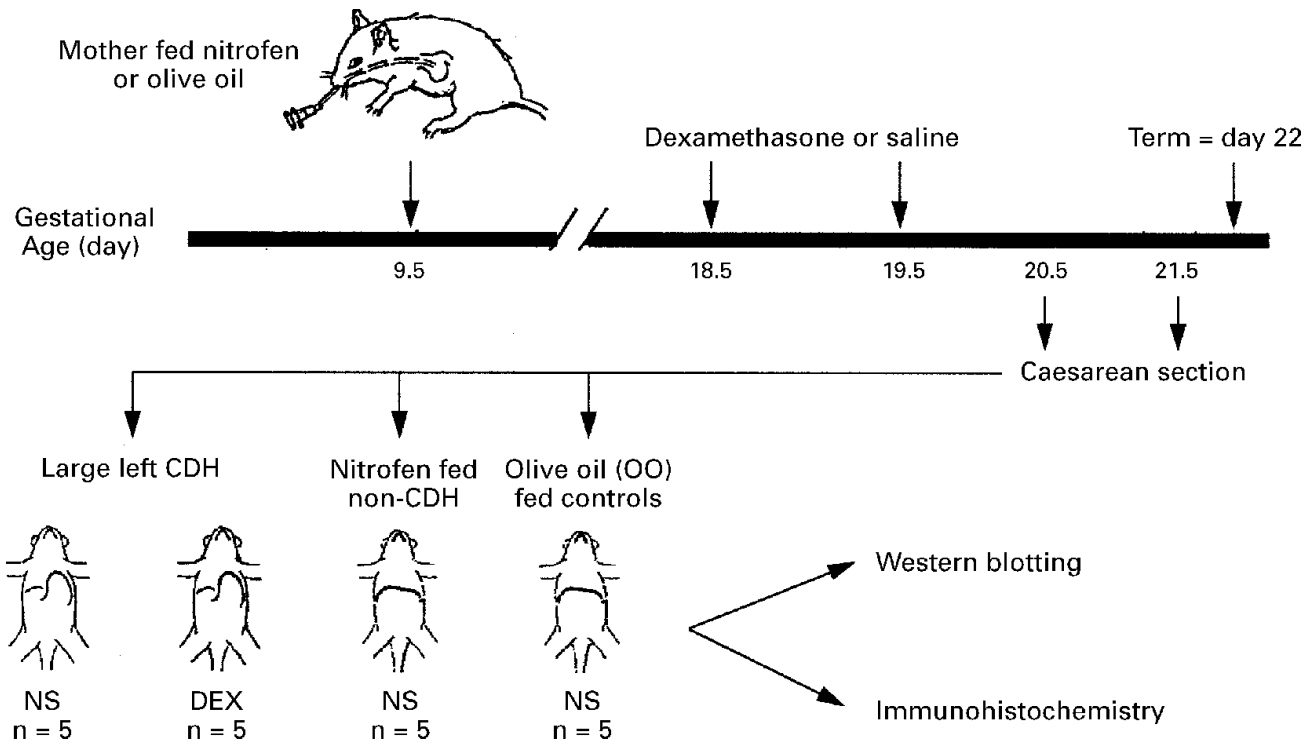

Figure 1 Experimental protocol: $N S=$ physiological saline, Dex $=$ dexamethasone, $O O=$ olive oil .

Antenatal dexamethasone ( David Bull Laboratories Warwick, UK) $0.25 \mathrm{mg} / \mathrm{kg}$ or an equal volume of physiological saline was administered by maternal intraperitoneal injection on days 18.5 and 19.5. Pregnant rats were terminally anaesthetised using halothane on days 20.5 and 21.5 of gestation. Fetal pups were given a lethal dose of sodium pentobarbital $100 \mathrm{mg} / \mathrm{kg}$ intraperitoneally to prevent air breathing and hypoxic stress before delivery by caesarean section. We studied the ipsilateral lungs as although the vascular abnormalities associated with $\mathrm{CDH}$ are known to be bilateral, they are more severe on the ipsilateral side. ${ }^{25}$ Alterations in the ipsilateral lung are therefore likely to be representative of events occurring in both lungs. ${ }^{26}$ Animals were divided into four experimental treatment groups (fig 1).

WESTERN IMMUNOBLOTTING

Individual lungs were stripped of all extra pulmonary tissue, flash frozen immediately in liquid nitrogen, and stored at $-70^{\circ} \mathrm{C}$ until they were studied. The lungs were homogenised in $10 \mathrm{mM}$ TRIS- $\mathrm{HCl}$ (pH 7.4) and $100 \mathrm{mM}$ $\mathrm{NaCl}$ and centrifuged at $5000 \times g$ for 3 minutes at $4^{\circ} \mathrm{C}$. Protein assays were performed on the supernatant fluid by the method of Lowry using bovine serum albumin to obtain standard curves. ${ }^{27}$ Lung samples were then boiled in lysis buffer containing $42 \mathrm{mM}$ TRIS ( $\mathrm{pH} 6.8$ ), 48 $\mathrm{mM}$ sodium dodecyl sulphate, $7 \%$ glycerol, and $5 \% \beta$-mercapto ethanol for 3 minutes. Lung protein $(100 \mu \mathrm{g})$ was loaded into individual wells on a $7.5 \%$ polyacrylamide gel, separated by sodium dodecyl-polyacrylamide gel electrophoresis and then electrophoretically transferred on to a polyvinyldiflouride membrane (ICN Pharm Ltd, Oxfordshire, UK). ${ }^{28}$ The membrane was then incubated with a solution of $5 \%$ non-fat milk in $10 \mathrm{mM}$ TRIS pH7.4, $100 \mathrm{mM} \mathrm{NaCl}$, and $0.1 \%$ Tween 20 (blocking buffer) for 1 hour to prevent non-specific binding of antibody, followed by an overnight incubation with a specific murine monoclonal antibody for eNOS (Transduction Laboratories, Kentucky, USA). After 6 five minute washes a 1 hour incubation with a secondary peroxidase labelled rabbit anti-mouse antibody (Sigma Aldrich Co. Dorset, UK) was performed. eNOS protein expression was visualised using a 1 minute incubation with chemiluminescent reagents (Amersham UK, Ltd.) and exposure to photo sensitive film (Amersham UK Ltd.). Western blots were quantified by laser densitometry using Image Quant software on a Personal Densitometer (Molecular Dynamics UK). eNOS expression for each specimen was reported as mean (SEM) percentage (compared with the eNOS expression in olive oil and physiological saline treated control lungs).

\section{IMMUNOHISTOCHEMISTRY}

Cryostat lung sections ( $8 \mu \mathrm{m}$ thick) were cut and the sections post-fixed in $1 \%$ formaldehyde for 1 minute, and acetone for 5 minutes. Sections were incubated with $2 \%$ bovine serum

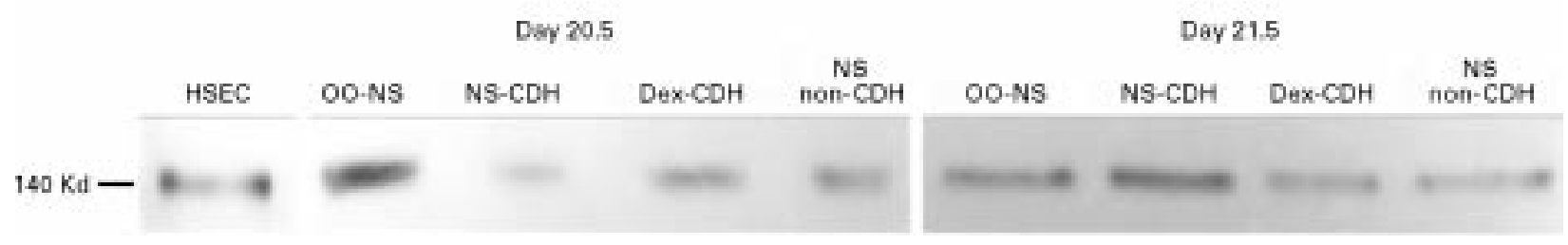

Figure 2 Western blots representative of five independent experiments showing pulmonary eNOS expression on days 20.5 and 21.5 of gestation: HSEC human suspended endothelial cells (positive control for eNOS). 
Pulmonary eNOS expression day 20.5
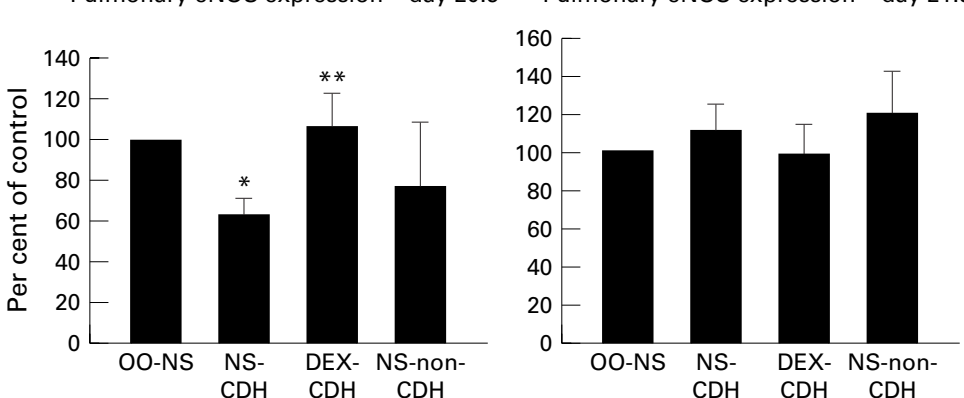

Figure 3 Results of five independent western blot experiments showing pulmonary eNOS expression on days 20.5 and 21.5 of gestation. Results are expressed as mean percentage (SEM) compared with eNOS expression in normal controls given olive oil and

physiological saline. On day 20.5 of gestation, $C D H$ rats given physiological saline showed reduced eNOS expression compared with normal controls (OO-NS) $\left({ }^{\star} p=0.03\right)$.CDH rats treated with dexamethasone had significantly higher eNOS concentrations than CDH fetuses given physiological saline $\left({ }^{\star *} p=0.02\right)$ and equivalent to that seen in the lungs of normal controls $(p=0.8)$.

albumin in TRIS buffer ( $\mathrm{pH}$ 7.6) to reduce non-specific binding of antibody and then with a specific murine monoclonal anti-eNOS antibody (Transduction Laboratories, Kentucky, USA) for 1 hour followed by two washes in TRIS buffer ( $\mathrm{pH}$ 7.6). This was followed by incubation with a secondary monoclonal biotin labelled rabbit anti mouse "link" immunoglobulin (Sigma Aldrich Co. Dorset, UK) and a streptavidin/horseradish peroxidase complex (DAKO Ltd. Bucks, UK). Staining for eNOS was visualised using a cobalt enhanced 3,3' diaminobenzidine chromogen (Sigma Aldrich Co. Dorset, UK). To confirm antibody specificity, control sections were subjected to all the above steps with omission of the primary anti eNOS antibody.

The unpaired $t$ test was used to determine statistical difference between the groups. A p value of $<0.05$ was considered significant.

\section{Results}

WESTERN IMMUNOBLOTTING

The results of five independent experiments and a representative western blot are shown in figs 2 and 3 for day 20.5. CDH lungs showed a significant reduction in eNOS protein expression compared with controls $(\mathrm{p}=0.03)$. In contrast, the lungs of $\mathrm{CDH}$ rats treated with dexamethasone showed eNOS expression equivalent to that seen in the lungs of normal animals $(\mathrm{p}=0.02$ vs $\mathrm{CDH}$ treated with physiological saline). Nitrofen physiological salinenon $\mathrm{CDH}$ pups had intermediate eNOS expression which were lower than but not statistically different from olive oil physiological saline normal controls $(p=0.07)$.

The results of five independent experiments and a representative western blot are shown in figs 2 and 3 for day 21.5. There were no differences in eNOS protein expression between any of the experimental groups studied.

\section{IMMUNOHISTOCHEMISTRY}

eNOS immunoreactivity was localised to the endothelium of pulmonary arteries of varying size in all the lungs studied (fig 4). Large hilar vessels showed the greatest intensity of staining compared with the smaller peripheral arterioles. There were no obvious differences in the intensity of staining between the groups. eNOS immunoreactivity was also detected in the bronchial epithelium, but no differences were observed between the experimental groups. Detailed morphometric analysis was not performed.

\section{Discussion}

Pulmonary hypertension in combination with pulmonary hypoplasia continues to present a major obstacle to the successful management of CDH. Despite advances in neonatal intensive care, the current mortality associated with this condition remains as high as $50 \% .{ }^{529}{ }^{30} \mathrm{An}$ improved understanding of the natural history and pathophysiology of $\mathrm{CDH}$ together with the availability of antenatal diagnosis has led to fetal intervention in an effort to rescue lung growth and maturation. Recently, antenatal glucocorticoid treatment has been shown to improve biochemical immaturity, increase lung compliance, and enhance lung morphology in experimental $\mathrm{CDH}$ animals. ${ }^{20-23}$ The effect of this treatment on the pulmonary vascular bed in $\mathrm{CDH}$ has not been studied.

In this study, we used the nitrofen induced $\mathrm{CDH}$ rat model which reproduces many of the pathologic hallmarks of $\mathrm{CDH}$ seen in people. The diaphragmatic defect is induced early in gestation during the embryonal phase of lung development producing lungs which are markedly hypoplastic, surfactant deficient, poorly compliant and display hypermuscularisation of the pulmonary vasculature. ${ }^{20-24} 3132$

Nitric oxide is believed to have a pivotal role during the transition of the fetal pulmonary circulation to extrauterine life. ${ }^{8}$ Experimentally, inhibiting NO production before birth can prevent the normal postnatal increase in pulmonary blood flow. ${ }^{83}$ Clinically, inhaled NO has been administered as a specific pulmonary vasodilator to $\mathrm{CDH}$ patients with varying clinical response. ${ }^{11-17}$ An eNOS deficiency in $\mathrm{CDH}$ has been investigated by previous authors. ${ }^{202634}$ eNOS activity is normal in CDH lambs, suggesting that a deficiency is not implicated in this late gestational animal model. ${ }^{34}$ In contrast, studies in $\mathrm{CDH}$ rats have yielded conflicting results. ${ }^{202635}$ In our study, we showed that eNOS was deficient in $\mathrm{CDH}$ rats at 20.5 days of gestation. This agrees with the findings of a previous study. ${ }^{26}$

We noted that dexamethasone treated $\mathrm{CDH}$ rats had eNOS concentrations restored to those seen in the lungs of normal olive oil controls. This suggests that antenatal steroids may enhance endothelium dependent vasorelaxation in fetal $\mathrm{CDH}$ rat lungs before term. However, at term (day 21.5 ), we were unable to show an absolute deficiency of eNOS, and dexamethasone had no additive effects on its expression. Supporting these findings, immunohistochemistry revealed no qualitative differences in eNOS immunoreactivity in the large and small pulmonary arteries between experimental groups. These findings therefore agree with those of another study which showed normal eNOS mRNA expression at 21.5 days in $\mathrm{CDH}$ rats. ${ }^{20} \mathrm{~A}$ fetal eNOS deficiency near term (day 20.5) may reflect an inadequate priming 

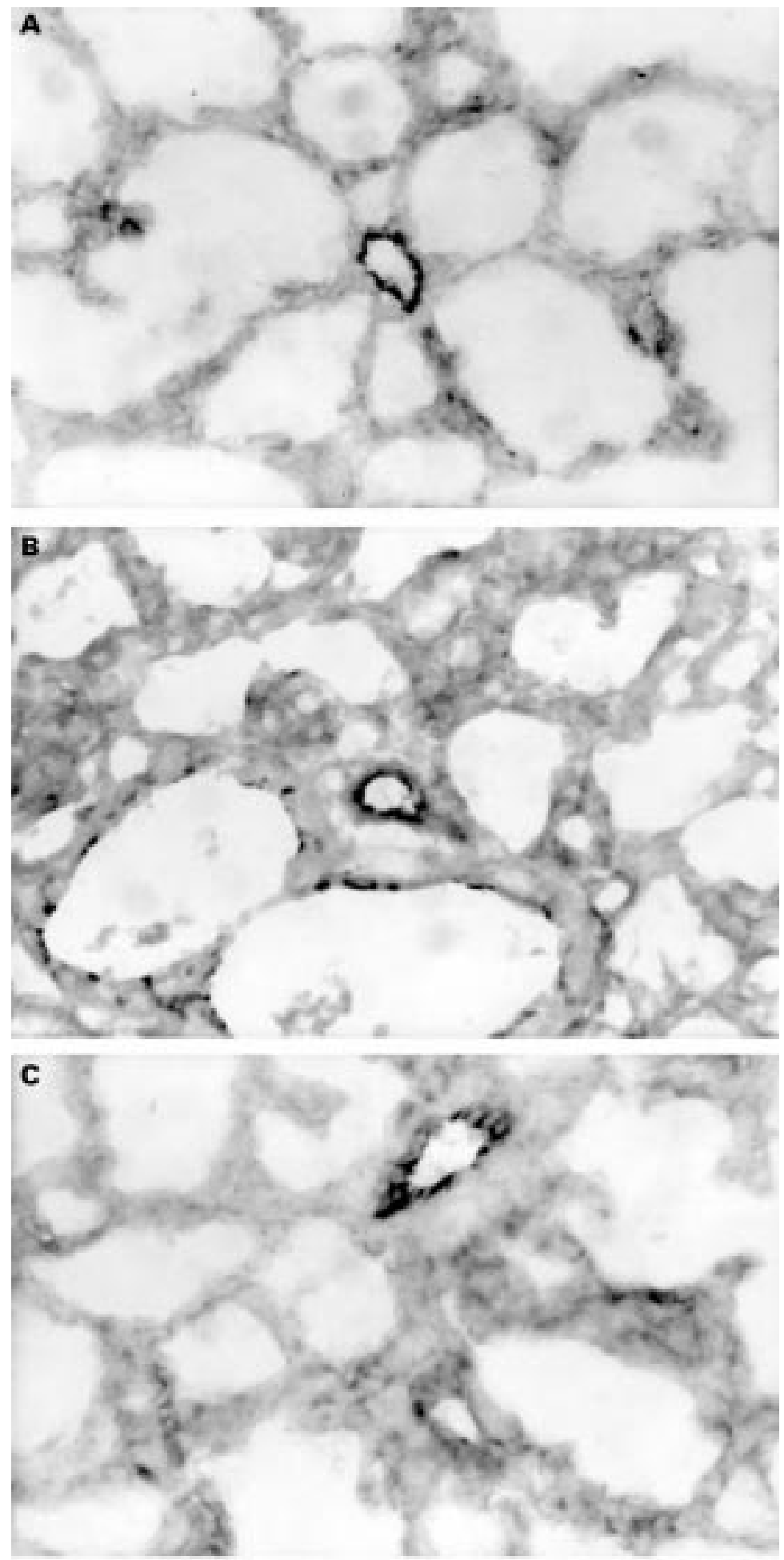

Figure 4 Photomicrographs showing eNOS immunoreactivity (black stain) in the endothelium of small pulmonary arteries in the experimental groups studied. eNOS immunoreactivity is also observed in the bronchial epithelium (B): small peripheral arteries of OO-NS (A); NS-CDH (B); and dexamethasone-CDH (C).

of the fetal pulmonary vascular bed for extrauterine life, as $\mathrm{NO}$ is known to have an important role in the regulation of fetal pulmonary vasoreactivity. ${ }^{7}$ By restoring eNOS expression at this time point, steroid treatment may improve the haemodynamic effects of fetal eNOS deficiency. However, at term the precise role of eNOS deficiency in $\mathrm{CDH}$ rats remains uncertain and this partly may explain the varied clinical response to exogenous NO treatment in people with $\mathrm{CDH} .{ }^{11-17}$ An imbalance or deficiency in eNOS may perhaps exist postnatally due to overwhelming vasoconstrictor influences and parenchymal $\mathrm{CDH}$ abnormalities. Antenatal glucocorticoids may address this imbalance by modulating vasoregulatory pathways and enhancing lung maturation. ${ }^{20-23} 3136$

Notably, we also showed eNOS immunoreactivity in the bronchial epithelium. This supports the findings of an earlier study that noted immunolocalisation within the ciliated bronchial epithelium of newborn rats. ${ }^{37}$ Thus NO may have an important role in modulating bronchial smooth muscle tone as well as vascular reactivity during the transition to neonatal life.

Glucocorticoids have profound maturational effects on the developing fetal lung. These effects include enhanced alveolar differentiation, thinning of alveolar septae and capillary walls, and upregulation of surfactant production. ${ }^{18} 38$ The mechanisms through which steroids produce these effects remain incompletely understood. They may occur as a result of the direct activation of glucocorticoid receptors or through the downstream paracrine or autocrine effects of growth factors..$^{39-42}$

The precise regulation of eNOS expression and activity has yet to be determined. eNOS gene expression can be upregulated by shear stress and hypoxia. ${ }^{43}$ Oestrogen may also upregulate eNOS expression through the activation of oestrogen receptors. ${ }^{44}$ Basic fibroblast growth factor and transforming growth factor $\beta 1$ can increase eNOS mRNA and protein expression. ${ }^{45}{ }^{46}$ Both growth factors can be susceptible to glucocorticoid modulation. ${ }^{47}{ }^{48}$ It is conceivable, therefore, that corticosteroids influence eNOS expression in the developing fetal lung which involves complex epithelial mesenchymal interactions. ${ }^{49}$ This process may be more complicated in pathological conditions such as $\mathrm{CDH}$ related lung hypoplasia where the mechanisms causing abnormal pulmonary growth and differentiation are incompletely understood.

In summary, antenatal glucocorticoid treatment can enhance the expression of eNOS in the lungs of fetal CDH rats near term. This could have implications for the therapeutic manipulation of fetal pulmonary vascular reactivity and may reduce the risk of pulmonary hypertension developing in the lungs of neonates with antenatally diagnosed CDH. Further studies are required to understand the regulatory mechanisms governing eNOS expression and NO activity in congenital diaphragmatic hernia.

This study was supported by grants from the Children's Research Fund and the Royal Liverpool Children's NHS Trust Endowment Fund, Liverpool.

1 Greenholz SK. Congenital diaphragmatic hernia : an overview. Semin Pediatr Surg 1996;5:216-23.

2 Collins DL, Pomerance JJ, Travis KW, et al. A new approach to congenital posterolateral diaphragmatic hernia. $\mathcal{f}$ Pediatr Surg 1977;12:149-56.

3 Kitigawa M, Hislop A, Boyden EA, et al. Lung hypoplasia in congenital diaphragmatic hernia. A quantitative study of airway, artery and alveolar development. $\mathrm{Br} f$ Surg $1971 ; 58: 342-6$ 
4 Nobuhara KK, Wilson JM. Pathophysiology of congenital diaphragmatic hernia. Semin Pediatr Surg 1996; 5:234-42.

van der Stak FHJM, de Haan AFJ, Geven WB, et al. Improving survival for high risk congenital diaphragmatic hernia by using extracorporeal membrane oxygenation. $f$ Pediatr Surg 1995;30:1463-7.

6 Rudolph AM. Fetal and neonatal pulmonary circulation. Ann Rev Physiol 1979;41:383-95.

7 Fineman JR, Soifer SJ, Heymann MA. Regulation of pulmonary vascular tone in the perinatal period. Ann Rev Physiol 1995;57:115-34.

8 Abman SH, Chatfield BA, Hall SL, et al. Role of endothelium derived relaxing factor during transition of pulmonary circulation at birth. Am f Physiol 1990;259 H1921-H7.

9 Steinhorn RH, Millard SL, Morin FC III. Persistent pulmonary hypertension of the newborn. Role of nitric oxide and endothelin in pathophysiology and treatment. Clin Perinatol 1995;22:405-28.

10 Forstermann U, Mulsch A, Bohme E, et al. Stimulation of soluble guanylate cyclase by an acetylcholine-induced
endothelium derived factor from rabbit and canine endothelium derived factor from

11 Shah N, Jacob T, Exler R, et al. Inhaled nitric oxide in congenital diaphragmatic hernia. F Pediatr Surg 1994;29:101015 .

12 Dillon PW, Cilley RE, Hudome SM, et al. Nitric oxide reversal of recurrent pulmonary hypertension and respiratory failure in an infant with CDH after successful ECMO therapy. F Pediatr Surg 1995;22:743-44.

13 Finer NN, Etches PC, Kamstra B, et al. Inhaled nitric oxide in infants referred for extracorporeal oxygenation: Dose response. F Pediatr 1994;124:302-8.

14 Karamanoukian HL Glick PL, Zayek M, et al. Inhaled nitric oxide in congenital hypoplasia of the lungs due to diaphragmatic hernia or oligohydramnios. Pediatrics 1994; 94:717-18.

15 Henneberg SW, Jepson S, Andersen PK, et al. Inhalation of nitric oxide as a treatment of pulmonary hypertension in
congenital diaphragmatic hernia. $\mathcal{f}$ Pediatr Surg congenital dias; $30: 853-5$.

16 Buhrer C, Merker G, Falke K, et al. Dose response to inhaled nitric oxide in acute hypoxaemic respiratory failure of newborn infants : a preliminary report. Pediatr 1995;19:291-8.

17 The neonatal inhaled nitric oxide study group ( NINOS) Inhaled nitric oxide and hypoxic respiratory failure in infants with congenital diaphragmatic hernia. Pediatrics 1997; 99:838-45.

18 Gross I. Regulation of fetal lung maturation. Am f Physiol 1990;259:L337-L44.

19 Crowley PA. Antenatal corticosteroid therapy: A metaanalysis of the randomised trials, 1972 to 1994 . Am f Obstet Gynecol 1995;173:322-35.

20 Suen HC, Bloch KD, Donahoe PK. Antenatal glucocorticoid treatment corrects the pulmonary immaturity of congenital diaphragmatic hernia. Pediatr Res 1994;35:523-9.

21 Losty PD, Suen HC, Manganaro TF, et al. Prenatal hormonal therapy improves pulmonary compliance in the nitro-
fen induced CDH rat model. 7 Pediatr Surg 1995;30:4206.

22 Losty PD, Pacheco BA, Manganaro TF, et al. Prenatal hormonal therapy improves pulmonary morphology in rats with congenital diaphragmatic hernia. I Surg Res 1996;65:42-52

23 Schnitzer JJ, Hedrick HL, Pacheco BA, et al. Prenatal glucocorticoid therapy reverses pulmonary immaturity in congenital diaphragmatic hernia in fetal sheep. Ann Surg 1996;224:430-9.

24 Kluth D, Kangah P, Reich P, et al. Nitrofen-induced diaphramatic hernias in rats: An animal model. 7 Pediat Surg 1990;25:850-4.

25 Geggel RL, Murphy JD, Langleben D, et al. Congenital diaphragmatic hernia: Arterial structural changes and persistent pulmonary hypertension after surgical repair. $\mathcal{f}$ sistent pulmonary hyperter
Pediatr 1985;107:457-64

26 North AJ, Moya FR, Mysore MR, et al. Pulmonary endothelial nitric oxide synthase gene expression is endothelial nitric oxide synthase gene expression is nia. Am 7 Respir Cell Mol Biol 1995;13: 676-82.

27 Lowry OH, Rosebrough NJ, Farr AL, et al. Protein measurement with the Folin phenol reagent. $f$ Biol Chem 1951;193: 265-75.
28 Laemmli UK. Cleavage of structural proteins during the assembly of the head of bacteriophage T4. Nature Lond 1970;227:680-5.

29 Sigalet DL, Tierney A, Adolph V, et al. Timing of repair of congenital diaphragmatic hernia requiring extracorporeal membrane oxygenation support. $\mathcal{f}$ Pediatr Surg 1995;30:1183-7.

30 Coughlin JP, Drucker DE, Cullen ML, et al. Delayed repair of congenital diaphragmatic hernia. Am Surg 1993;58:90-3.

31 Okoye BO, Losty PD, Lloyd DA, et al. The effect of prenatal glucocorticoids on pulmonary vascular muscularisation in rats with nitrofen-induced congenital diaphragmatic hernia. F Pediatr Surg. 1998;33:76-80

32 Tenbrinck R, Gaillard JLJ, Tibboel D, et al. Pulmonary vascular abnormalities in experimentally induced congenital diaphragmatic hernia in rats. 7 Pediatr Surg 1992;27:862-5.

33 Fineman JR, Wong J, Morin IFC, et al. Chronic nitric oxide inhibition in utero produces persistent pulmonary hypertension in newborn lambs. F Clin Invest 1994;93:2675-83.

34 O'Toole SJ, Irish MS, Holm BA, et al. Pulmonary vascular abnormalities in congenital

35 Karamanoukian HL, Peay T, Love JE, et al. Decreased pulmonary nitric oxide synthase activity in the rat model of congenital diaphragmatic hernia. $f$ Pediatr Surg 1996;31:1016-19.

36 Okoye BO, Losty PD, Fisher MJ, et al. Antenatal glucocorticoid therapy suppresses angiotensin-converting enzyme activity in rats with nitrofen induced congenital diaphragmatic hernia. F Pediatr Surg 1998;33:286-91.

37 Xue C, Botkin SJ, Johns RA. Localization of endothelial NOS at the basal microtubule membrane in ciliated epithelium of rat lung. F Histochem Cytochem 1996;44:463-

38 Vyas J, Kotecha S. Effects of antenatal and postnatal corticosteroids on the preterm lung. Arch Dis Child 1997;77:F147-F50.

39 Gross I, Dynia DW, Rooney SA, et al. Corticosteroid stimulation of phosphatidylcholine synthesis in cultured fetal rabbit lung. Evidence for denovo protein synthesis mediated by glucocorticoid receptors. Endocrinology 1983;112:829-37.

40 Wang J, Kuliszewski M, Yee W, et al. Cloning and expression of glucocorticoid -induced genes in fetal rat lung fibroblasts. Transforming growth factor, beta 3. F Biol Chem 1995;270:2722-8.

41 Sadiq HF, Devaskar UP. Glucocorticoids increase pulmonary epidermal growth factor receptors in female and male fetal rabbit. Biochem Biophys Res Commun 1984;119:40814 .

42 Mouhieddine OB, Cazali V, Kuto E, et al. Glucocorticoid induced growth arrest of lung alveolar epithelial cells is associated with increased production of insulin-like growth factor binding protein 2. Endocrinol 1996;137:28795

43 Nathan C, Xie Q. Regulation and biosynthesis of NO. F Biol Chem 1994;269:13725-8.

44 MacRitchie AN, Jun SS, Chen Z, et al. Estrogen upregulates endothelial nitric oxide synthase gene expression in fetal pulmonary artery endothelium. Circ Res 1997;81:355-62.

45 Kostyk SK, Kourembanas S, Wheeler EL, et al. Basic fibroblast growth factor increases nitric oxide synthase production in bovine endothelial cells. Am $\mathcal{f}$ Physiol 1995;269:H1583- 9.

46 Inoue N, Venema RC, Sayegh HS. Molecular regulation of the bovine endothelial nitric oxide synthase by transforming growth factor -beta 1. Art Thromb Vasc Biol $1995 ; 15: 1255-61$.

47 Meisinger C, Zeschnigk C, Grothe C. In vivo and in vitro effect of glucocorticoids on fibroblast growth factor (FGF)-2 and FGF receptor 1 expression. 7 Biol Chem 1996;271:16520-5.

48 Jaskoll T, Choy HA, Melnic M. The glucocorticoidglucocorticoid receptor signal transduction pathway, transforming growth factor $\beta$, and embryonic mouse lung development in vivo. Pediatr Res 1996;39:749-59.

49 Minoo P, King RJ. Epithelial-mesenchymal interactions in lung development. Annu Rev Physiol 1994;56:13-45. 